\title{
Speckle interferometry of the HAeBe star V376 Cas ${ }^{\star}$
}

\author{
K. W. Smith ${ }^{1}$, Y. Y. Balega ${ }^{2}$, K.-H. Hofmann ${ }^{1}$, Th. Preibisch ${ }^{1}$, D. Schertl ${ }^{1}$, and G. Weigelt ${ }^{1}$ \\ 1 Max-Planck-Institut für Radioastronomie (MPIfR), Auf dem Hügel 69, 53121 Bonn, Germany \\ ${ }^{2}$ Special Astrophysical Observatory, Nizhnij Arkhyz, Zelenchuk region, Karachai-Cherkesia 357147, Russia
}

Received 11 June 2003 / Accepted 18 September 2003

\begin{abstract}
We report $H$ - and $K^{\prime}$-band speckle interferometric observations of the HAeBe star V376 Cas. Our observations show that the object is partially resolved. The visibility curves suggest three separate components: a large scattering envelope visible only in the $H$ band, a component approximately 100 mas in radius, and a component with a Gaussian HWHM of approximately $8 \pm 3$ mas, corresponding to approximately $5 \mathrm{AU}$ at $600 \mathrm{pc}$ distance, which contributes most of the flux. We compare the smallest structure to the radius of dust sublimation in the radiation field of the star and find that the radius is approximately six times larger than that expected. This may indicate that the inner regions of the system are in fact obscured by a flaring circumstellar disk or torus seen close to edge-on.
\end{abstract}

Key words. stars: circumstellar matter - stars: formation - stars: individual: V376 Cas

\section{Introduction}

Many intermediate-mass young stellar objects (YSOs) are surrounded by large quantities of circumstellar material, giving rise to infrared excesses which often dominate their spectra. Much of this material is often distributed in a circumstellar disk, through which accretion onto the central object may continue. Whilst simple disk models originally explained many of the features of YSOs, there remained some outstanding problems with a thin disk picture. Principally, for both flat passive reprocessing disks and active accretion disks, $\lambda F_{\lambda} \propto \lambda^{-4 / 3}$ (Lynden-Bell \& Pringle 1974; Adams et al. 1987) whereas the observed spectral energy distributions (SEDs) are in general flatter than this. This implies that either a modification of the disk model or the presence of some other component to the circumstellar material is necessary.

Hillenbrand et al. (1992) examined the SEDs of a large sample of HAeBe stars and divided them into three groups; those which could be modelled with a geometrically thin disk, sometimes requiring an optically thin inner hole, those with a rising SED at long wavelengths, which could be modelled only with an envelope, and those which lacked either disks or envelopes. These categories were suggested as an evolutionary scheme for circumstellar environments of HAeBe stars. Hartmann et al. (1993) suggested instead that a combination of disks and envelopes was generally necessary. Since then, disk+envelope models have been further investigated by

Send offprint requests to: K. Smith, e-mail: kester@mpifr-bonn.mpg.de

* Based on observations performed with the $6 \mathrm{~m}$ telescope of the Special Astrophysical Observatory, Russia. several authors, including for example Natta et al. (1993), Pezzuto et al. (1997), and Miroshnichenko et al. (1997, 1999).

The presence of disks together with envelopes is strongly suggested by the observations of Mannings \& Sargent (1997, 2000) who studied several intermediate mass YSOs in the millimetre continuum and various molecular lines. For several sources, they found line profiles consistent with Keplerian rotation. In some cases the rotation curve could be spatially resolved. The quantity of material inferred from millimetre continuum fluxes was generally not consistent with measured extinctions and a spherically symmetric distribution.

The main alternative to disk+envelope models is some type of flared disk (e.g. Chiang \& Goldreich 1997). For a reprocessing disk, such models modify the temperature power law and flatten the SED at far-infrared (FIR) wavelengths. Malbet et al. (2001) and Lachaume et al. (2003) considered flared-disk models in which the disk is heated by both stellar light and viscous dissipation and had success in fitting SEDs. However, simple flared-disk models cannot reproduce the near-infrared (NIR) "bump" between about 1 and $3 \mu \mathrm{m}$ observed in many HAeBe systems. Dullemond et al. (2001) and Natta et al. (2001) therefore proposed a new variant of the flared-disk model, introducing an inner hole and thereby producing a bright inner rim which contributes more NIR flux than a simple flared disk. This inner rim can puff up and partially or completely shadow the more distant disk. Beyond any shadowed region, the outermost regions of the disk are the same as envisaged in the simple flared-disk picture, maintaining the flux at FIR wavelengths.

In recent years, advances in speckle observing techniques, the advent of adaptive optics, and NIR long-baseline interferometry have begun to allow insights into the spatial 
distribution of the circumstellar material and outflows associated with YSOs. For example, Leinert et al. (2001) used a speckle technique to observe $31 \mathrm{HAeBe}$ stars and found many to have envelopes. The complex environment of the HAeBe star R Mon was studied by Close et al. (1997) with adaptive optics and by Weigelt et al. (2002a) with bispectrum speckle interferometry, revealing several structures related to the disk and outflow. Similar bispectrum speckle observations of the highmass YSOs S140 IRS 1 by Schertl et al. (2000) and Weigelt et al. (2002b) and of S140 IRS 3 by Preibisch et al. (2001) revealed complex outflows associated with these objects. Monin $\&$ Bouvier (2000) used an adaptive optics system with a resolution of about 70 mas in the $J$ band to image an edge-on circumstellar disk in the young triple system HV Tau. Danchi et al. (2001) imaged the massive young star MWC 349A using aperture-masking interferometry and found what appeared to be an edge-on disk. This object was also observed by Hofmann et al. (2002) who used bispectrum speckle interferometry and also saw the same probable edge-on disk. Other edge-on disks have been imaged using HST (see for example McCaughrean \& O'Dell 1996 or Brandner et al. 2000). Tuthill et al. (2002) used an aperture masking interferometry technique to resolve the massive $\mathrm{HAeBe}$ star $\mathrm{LkH} \alpha 101$ and saw a circular object about 50 mas across, corresponding to $3.4 \mathrm{AU}$ at $160 \mathrm{pc}$. This object showed an asymmetry reminiscent of an almost face-on but marginally inclined disk. Malbet et al. (1998) resolved FU Ori with the Palomar Testbed Interferometer (PTI). Akeson et al. (2000) observed four YSOs with the PTI and resolved three of them, two T Tauri stars and an HAeBe star. Millan-Gabet et al. (2001) made interferometric observations of a sample of fifteen HAeBe stars and saw many resolved sources. Typical sizes were a few milliarcseconds, corresponding to between about 0.5 and a few AU. Some of the above results were surprising, since the hot regions of classical accretion disk models, from where the NIR flux should largely arise, should be too small to be resolved at this level.

V376 Cas is a HAeBe star embedded in the small dark cloud L1265 and associated with considerable nebulosity. At optical wavelengths, on arcsecond scales, this nebulosity is seen to be elongated along an axis with position angle approximately $120^{\circ}$ (Leinert et al. 1991; Piirola et al. 1992; Corcoran et al. 1995; Asselin et al. 1996). The source displays an extreme degree of linear polarization in the red, $\sim 20-25 \%$, higher than for any other intermediate mass YSO, indicating that a high proportion of the emerging light has been scattered. Polarization maps were produced at $900 \mathrm{~nm}$ by Leinert et al. (1991), in the $I$ band by Piirola et al. (1992), and in the optical by Asselin et al. (1996). These maps show strong centrosymmetric polarization in the lobes of the elongated nebulosity and areas of low polarization situated near the star on either side of the extended envelope. Leinert et al. (1991) used speckle polarimetry at $K$ to show that this pattern persists to subarcsecond scales. This pattern of polarization suggests a bipolar reflection nebula aligned northwest-southeast, which in turn implies the presence of an edge-on disk at a position angle of approximately $30^{\circ}$. HST WFPC2 images obtained from the archive (Fig. 1) confirm this elongated source. However, at this higher resolution, the overall nebulosity is not reminiscent of an edge-on disk with position angle $30^{\circ}$, but rather suggests an outflow cavity to the west of the object. This in turn suggests a nearly edge-on disk at position angle approximately $140^{\circ}$, together with the nearside lobe of a bipolar outflow extending to the west. The observed light probably arises mostly from scattering from the inner edge of the outflow cavity or from the surface of a flared disk or circumstellar torus.

\section{Observations and data reduction}

The speckle interferograms were recorded in September 2002 with the SAO $6 \mathrm{~m}$ telescope in Russia. The detector of the speckle camera was a Rockwell HAWAII array. The two filters used corresponded approximately to $H$ (central wavelength $1.648 \mu \mathrm{m}$, bandwidth $0.317 \mu \mathrm{m}$ ) and $K^{\prime}$ (central wavelength $2.115 \mu \mathrm{m}$, bandwidth $0.214 \mu \mathrm{m}$ ). Further observational parameters are listed in Table 1.

The modulus of the object Fourier transform (visibility) was obtained with the speckle interferometry method (Labeyrie 1970). Speckle interferograms of calibrator stars, which were measured to be unresolved, were recorded just before and just after the object and served as reference stars for the determination of the speckle transfer function (see Table 1).

\section{Results and discussion}

The reconstructed visibilities of V376 Cas are presented in Fig. 2. The upper two panels show the two-dimensional visibilities in $K^{\prime}$ (left) and $H$ (right). The visibilities show some evidence of elongation along a position angle approximately $70^{\circ}$ from the vertical. The $H$-band visibility is dominated by a central spike, indicating the extended emission in the $3.8^{\prime \prime}$ field of view of $H$, which is not evident at $K^{\prime}$. The azimuthally averaged visibilities are shown in the two lower panels of Fig. 2. These plots have both been truncated at twelve cycles per arcsecond, beyond which the quality of the data is significantly degraded by noise. There is clear evidence of resolution at both wavelengths. The $K^{\prime}$-band visibilities decline by about $20 \%$ and the $H$-band visibilities by about $30 \%$ over the range of spatial frequencies sampled. Both curves show a significant decrease in steepness at $\sim 4$ cycles/arcsec. This structure suggests a two-component visibility model for the $K^{\prime}$ band and a threecomponent visibility model for the $H$ band. The best-fitting 3and 2-component Gaussian models for $H$ and $K^{\prime}$ are shown in the plots. A second model was also fitted, in which the most compact Gaussian component (on the sky) is replaced by a uniform-brightness ring. The inner radius of the ring was the fitted parameter, and the outer radius was set at $110 \%$ of this. The intention of fitting this model is to allow direct comparison with the results reported for similar objects in the literature (e.g. Millan-Gabet et al. 2001). To determine uncertainties in the fitted parameters, each individual target-reference star pair was fitted separately and the resulting parameters averaged. There were five such independent observations in total in each band. The peak-to-peak variation of each parameter was then used as an estimate of the uncertainty. Table 2 lists the parameters and associated uncertainties of all these fits. 


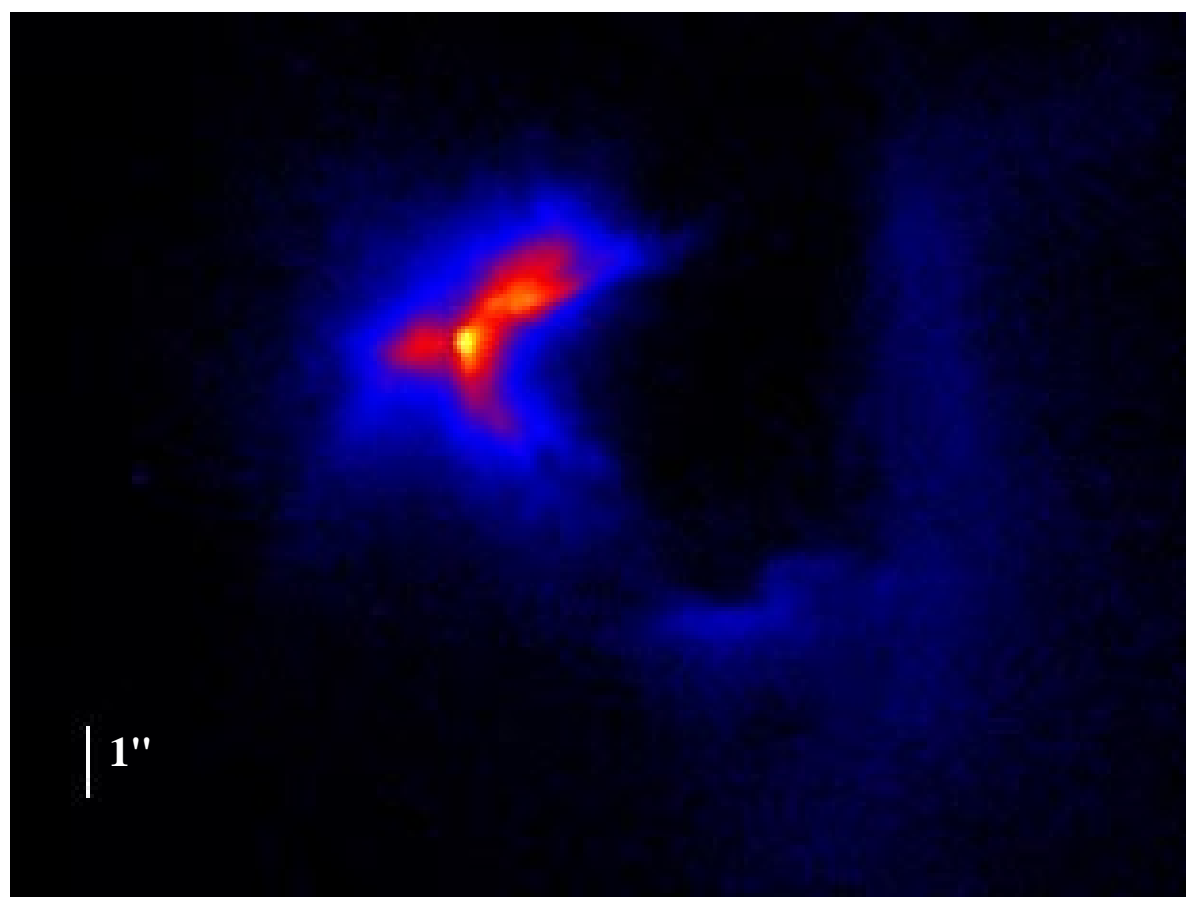

Fig. 1. HST WFPC2 image of V376 Cas taken with the $\mathrm{F} 814 \mathrm{~W}$ filter at $800 \mathrm{~nm}$. This image, which was obtained from the archive, is the combination of one $100 \mathrm{~s}$ and one $300 \mathrm{~s}$ exposure, both of which were made in March 2000. North is up and East is to the left. The brightest knot probably corresponds to the component observed at $H$ and $K^{\prime}$.

Table 1. Observational parameters. $\lambda_{\mathrm{c}}$ and $\Delta \lambda$ give the central wavelength and bandwidth of the filter. FoV is the field of view of both the data recording and data processing. $N_{\mathrm{T}}$ and $N_{\mathrm{R}}$ are the total numbers of speckle interferograms of V376 Cas and the reference star, respectively, and $N_{\mathrm{P}}$ is the number of target-object pairs observed per night. $T$ is the exposure time per frame. In the last column, the names of the reference stars are given.

\begin{tabular}{llllllllll}
\hline \hline $\begin{array}{l}\text { Observation Day } \\
\text { Sep. 2002 }\end{array}$ & $\begin{array}{l}\lambda_{\mathrm{c}} \\
{[\mathrm{nm}]}\end{array}$ & $\begin{array}{l}\Delta \lambda \\
{[\mathrm{nm}]}\end{array}$ & $\begin{array}{l}\text { FoV } \\
\left({ }^{\prime \prime}\right)\end{array}$ & $N_{\mathrm{T}}$ & $N_{\mathrm{R}}$ & $N_{\mathrm{P}}$ & $\begin{array}{l}T \\
{[\mathrm{~ms}]}\end{array}$ & $\begin{array}{l}\text { Seeing } \\
{\left[{ }^{\prime}\right]}\end{array}$ & $\begin{array}{l}\text { Reference } \\
\text { Star }\end{array}$ \\
\hline 20 & 2115 & 214 & 5.2 & 562 & 732 & 1 & 164 & 2.2 & HD 236352 \\
22 & 2115 & 214 & 5.2 & 275 & 536 & 1 & 245 & 3.2 & HD 236352 \\
23 & 2115 & 214 & 5.2 & 368 & 750 & 1 & 164 & 2.2 & HD 236352 \\
25 & 2115 & 214 & 5.2 & 635 & 1264 & 2 & 184 & 2.1 & HD 236324 \\
\hline 24 & 1648 & 317 & 3.8 & 2083 & 3160 & 5 & 180 & 1.7 & HD 236324 \\
\hline
\end{tabular}

The Gaussian fit components are an approximately $1^{\prime \prime}$ HWHM extended nebula visible only in $H$, an approximately 100 mas HWHM component, corresponding to about $70 \mathrm{AU}$ in radius at an assumed distance of $600 \mathrm{pc}$ (Chavarria-K 1985), and a small, approximately $8 \pm 3$ mas HWHM component comprising a large fraction of the flux in each band, corresponding to a region of about $4.8 \mathrm{AU}$ in radius at $600 \mathrm{pc}$ (see Fig. 2). It should be stressed that smaller components could in fact be present which are not resolved at our longest baselines. We cannot, of course, exclude the possibility of another break in the visibility curve at baselines longer than $6 \mathrm{~m}$.

The $H$-band visibility function shows resolved structure both along and across the elongation. This, together with the approximately circularly symmetric appearance of the $K^{\prime}$-band visibility function allows us to rule out a binary model as the sole explanation for the resolved source and to identify at least part of the resolved structure with circumstellar material. It is possible that a companion is also present, with a position angle of approximately $160^{\circ}$ or $340^{\circ}$, and contributes partially to the resolved structure. It is also possible that the elongation is due to the presence of a nearly edge-on disk. If this were so, the disk plane would run along a direction perpendicular to the observed elongation in the visibility function, that is, from northwest to southeast. Such a disk would be broadly compatible with the interpretation offered above for the HST image, showing an outflow cavity extending to the southwest.

\subsection{The 8 mas component: Dust sublimation?}

An explanation often found in the literature for the observed NIR sizes of YSOs is that the observed structure lies at the dust sublimation radius, that is, the radius at which circumstellar dust sublimates due to the radiation field of the central star. This gives rise either to a hot inner edge of a disk, as in the model of Dullemond et al. (2001) and similar models, or to a sharp inner boundary to a spherically symmetric shell. This type of explanation was first suggested for individual objects by Tuthill et al. (2001) and Natta et al. (2001), and was investigated for a sample of resolved YSOs by Monnier \& Millan-Gabet (2002). Monnier \& Millan-Gabet 

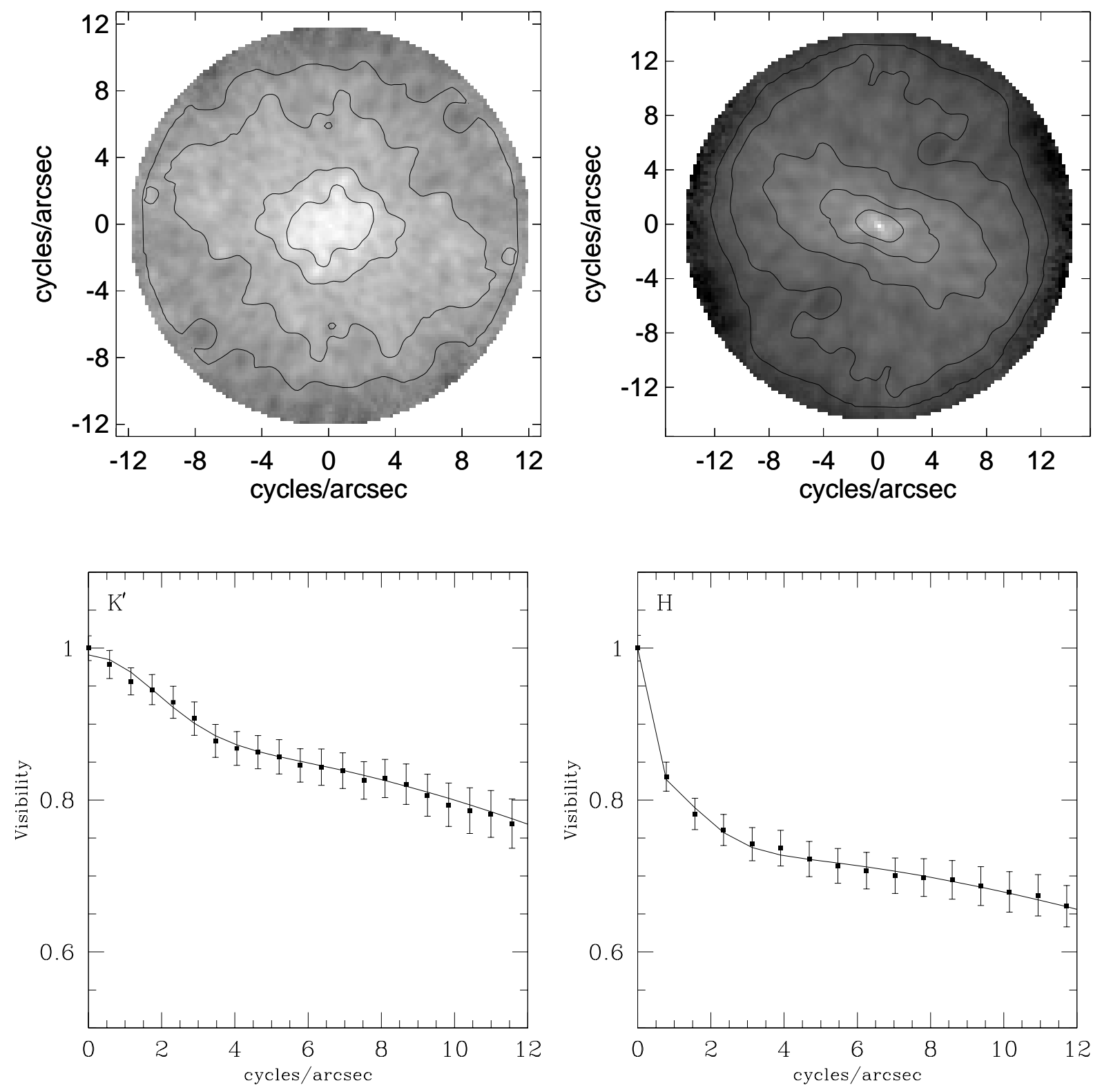

Fig. 2. Upper panels: two-dimensional visibilities of V376 Cas. Left column filter 2115/214 nm. Right column filter $1648 / 317 \mathrm{~nm}$. The contours are drawn at levels 0.95, 0.90, 0.85, 0.80, 0.75 and 0.70. Lower panels: Azimuthally averaged visibilites. Left column filter 2115/214 nm. Right column filter $1648 / 317 \mathrm{~nm}$. The individual dots show the observed visibilities, the solid line the fitted model consisting of two Gaussians at $2115 \mathrm{~nm}$ and three Gaussians at $1648 \mathrm{~nm}$.

(2002) demonstrated that the NIR sizes of various HAeBe and $\mathrm{T}$ Tauri stars appearing in the literature correlates with the stellar luminosity in a way that is consistent with their being determined by dust sublimation. Here, we compare the smallest resolved component of V376 Cas with the expected dust sublimation radius for this object.

The dust sublimation radius can be estimated as

$$
\begin{aligned}
R_{\mathrm{S}} & =\frac{1}{2} \sqrt{Q_{R}}\left(\frac{T_{*}}{T_{\mathrm{s}}}\right)^{2} R_{*} \\
& =1.1 \sqrt{Q_{R}}\left(\frac{L_{*}}{1000 L_{\odot}}\right)^{1 / 2}\left(\frac{T_{\mathrm{s}}}{1500 \mathrm{~K}}\right)^{-2} \mathrm{AU},
\end{aligned}
$$

where $Q_{R}$ is the ratio of the incident absorption efficiency to the re-emitted absorption efficiency (Monnier \& Millan-Gabet 2002).

For hot stars, $Q_{R}$ can vary strongly if the grain size is smaller than about one micron. For grains of $1 \mu \mathrm{m}$ or larger, $Q_{R} \sim 1$, but $Q_{R}$ may reach values of around 50 or more for grain sizes of less than $0.25 \mu \mathrm{m}$ and stellar surface temperatures of $10000 \mathrm{~K}$ or greater. However, if large grains existed in sufficient quantities, they would survive longer and determine the position of $R_{\mathrm{s}}$. This logic was followed by both Monnier $\&$ Millan-Gabet (2002) and Tuthill et al. (2001), who both assumed $Q_{R}=1$. 
Table 2. Results of circular symmetric fits to the observed visibilities. Two different models were fitted at each wavelength. The first model consisted of either two (at $K^{\prime}$ ) or three (at $H$ ) Gaussians. For the second model, the broadest Gaussian in the visibilities (corresponding to the narrowest component on the sky) was replaced with a uniform-brightness ring, the inner radius of which was a fitted parameter and the outer radius of which was set to $110 \%$ of the inner radius.

\begin{tabular}{lllllll}
\hline \hline \multicolumn{7}{c}{ Multiple Gaussian models } \\
\hline Small Component & \multicolumn{2}{c}{ Medium Component } & \multicolumn{2}{c}{ Large Component } \\
Filter & HWHM & Relative & HWHM & Relative & HWHM & Relative \\
{$[\mathrm{nm}]$} & {$[\mathrm{mas}]$} & flux & {$[$ mas] } & flux & {$[$ mas] } & flux \\
\hline 2115 & $8.2 \pm 3$ & $88 \pm 4 \%$ & $105 \pm 50$ & $11 \pm 5 \%$ & - & - \\
1648 & $7.4 \pm 3$ & $73 \pm 3 \%$ & $134 \pm 50$ & $11 \pm 5 \%$ & $711_{-350}^{+700}$ & $16 \pm 4 \%$ \\
\hline
\end{tabular}

Uniform-brightness ring plus Gaussian models

\begin{tabular}{lllllll}
\hline & \multicolumn{2}{c}{ Small Component } & \multicolumn{2}{c}{ Medium Component } & \multicolumn{2}{c}{ Large Component } \\
Filter & Ring inner & Relative & HWHM & Relative & HWHM & Relative \\
{$[\mathrm{nm}]$} & radius [mas] & flux & [mas] & flux & [mas] & flux \\
\hline 2115 & $9.6 \pm 3$ & $88 \pm 4 \%$ & $104 \pm 50$ & $10.4 \pm 4 \%$ & - & - \\
1648 & $8.3 \pm 3$ & $73 \pm 4 \%$ & $124 \pm 80$ & $10.9 \pm 4 \%$ & $843 \pm 700$ & $16.6 \pm 3 \%$ \\
\hline
\end{tabular}

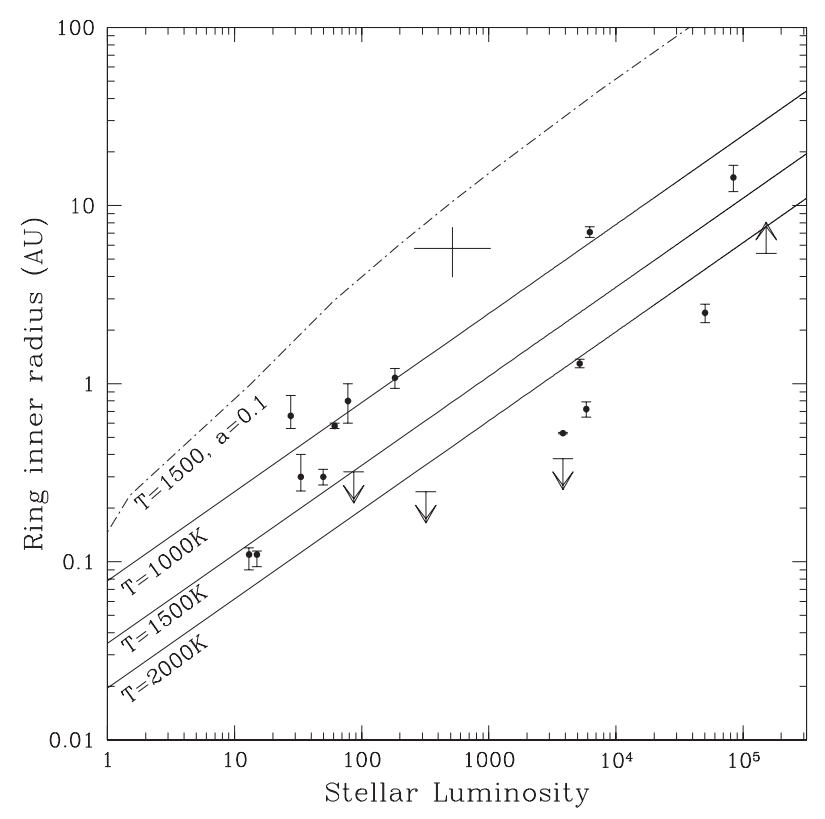

Fig. 3. The sizes of YSOs as a function of stellar luminosity, based on Fig. 1 of Monnier \& Millan-Gabet (2002). The data points show the inner ring radii of uniform-ring fits to resolved sources from Millan-Gabet et al. (2001), the measured size of $\mathrm{LkH} \alpha 101$ from Tuthill et al. (2002), and the HWHM for Gaussian fits to two classical $\mathrm{T}$ Tauri stars from Akeson et al. (2000). The cross marks the position of V376 Cas. The luminosity has been taken as $517 L_{\odot}$ with an assumed error of a factor of two. The radius plotted is the inner ring radius of the smallest component fitted to the $K^{\prime}$ data. The straight lines mark the model with $Q_{R}=1$ for three dust sublimation temperatures, $1000 \mathrm{~K}, 1500 \mathrm{~K}$, and $2000 \mathrm{~K}$. The dashed line is a model with a grain size of $0.1 \mu \mathrm{m}$ and $T_{\mathrm{s}}=1500 \mathrm{~K}$.

The maximum temperature at which dust grains can survive is estimated to be around $1750 \mathrm{~K}$ for graphite and about $1400 \mathrm{~K}$ for silicate grains (Hillenbrand et al. 1992). We use a sublimation temperature of $1500 \mathrm{~K}$ but also consider temperatures of $1000 \mathrm{~K}$ and $2000 \mathrm{~K}$.
Various estimates of the luminosity of V376 Cas are available in the literature. Chavarria-K. (1985) estimated the luminosity of V376 Cas to be $517 L_{\odot}$, based on his own extinction and distance estimate and the photometry of Allen (1972) and Cohen (1974). Ábrahám et al. (2000) observed V376 in the mid to far infrared with ISOPHOT and derived a luminosity between 1 and 1000 microns of $430 L_{\odot}$. Since the vast majority of the flux emerges in this wavelength range, this value is probably not a serious underestimate due to the wavelength truncation at either end. Natta et al. (1992) estimated the luminosity of V376 Cas to be about $150 L_{\odot}$, based on a combination of new FIR observations and existing optical and NIR measurements. For the purposes of this analysis, we adopt the luminosity of $517 L_{\odot}$ given by Ábrahám et al. (2000), together with an uncertainty of a factor of two.

With these assumptions $\left(L_{*}=517 L_{\odot}, T_{\mathrm{s}}=1500 \mathrm{~K}, Q_{R}=\right.$ 1) the dust sublimation radius for V376 Cas given by Eq. (1) is $0.79 \mathrm{AU}$. This rises to $1.78 \mathrm{AU}$ if the dust is destroyed at a temperature of $1000 \mathrm{~K}$. These values lie approximately a factor of three to six below the HWHM of the smallest fitted Gaussian for V376 Cas, at about 4.8 AU. This is illustrated in Fig. 3, which is based in Fig. 1 of Monnier \& Millan-Gabet (2002). This plot shows the relationship between stellar luminosity and dust sublimation radius for dust grains of $1 \mu \mathrm{m}$ or larger and three different dust sublimation temperatures, as well as the dust sublimation radius for a dust destruction temperature of $1500 \mathrm{~K}$ and a grain size of $0.1 \mu \mathrm{m}$. The position of V376 Cas in the $K^{\prime}$ band is marked. The error bars in the size are determined from the fitting procedure, as described in Sect. 3 above. The luminosity is assumed to be uncertain by a factor of two. No account is taken of possible errors in the distance of $600 \mathrm{pc}$. This is because an error in the distance would move the point parallel to the model lines in the figure and would therefore have no effect on any comparison between the data point and the models. Also shown are points for a number of other HAeBe and $\mathrm{T}$ Tauri stars taken from the literature (see caption for references). 


\subsubsection{Comparison with models}

The most compact component of V376 Cas which we resolve is about six times larger than the basic dust sublimation model predicts and also appears large compared to the population of similar objects shown in Fig. 3. Two possible explanations for this are that the dust grain population is dominated by grains significantly smaller than $1 \mu \mathrm{m}$, and that the inner regions of the system are obscured by a disk or torus viewed close to edge-on.

In Fig. 3 we show the estimated dust sublimation radius for grains of $0.1 \mu \mathrm{m}$ in size. Such a grain size leads to a predicted dust sublimation radius significantly larger than the measured size of V376 Cas, demonstrating that even a modest change in grain properties might accommodate our result. The scattering envelope seen at $H$ but not at $K^{\prime}$ may indicate that the scattering cross section is very much smaller at $K^{\prime}$ than at $H$. This could imply that the population of dust grains in this extended region is dominated by small grains. However, the position of V376 Cas in Fig. 3 is not only somewhat high compared to the models but also compared to other similar objects. Invoking a small dust-grain size to explain this would therefore involve suggesting that V376 Cas is in this respect an exceptional case.

Can the luminosity estimate for V376 Cas be in error? The spectral type of V376 Cas was determined by Cohen \& Kuhi (1979) from low resolution spectra to be B5. Earlier, Herbig (1960) estimated the spectral type of V376 Cas to be late B or early A. Rodriguez \& Canto (1983) observed the field at $6 \mathrm{~cm}$ and saw no corresponding radio source down to a level of approximately $1 \mathrm{mJy}$, which implies that the spectral type cannot be much earlier than mid B. Comparison with evolutionary tracks (e.g. Palla \& Stahler 1993; Driebe 2001) suggests that a B5 star of surface temperature $14000 \mathrm{~K}$ should have luminosity of between approximately 200 and $1000 L_{\odot}$. This is broadly consistent with the Chavarria-K. (1985) and Ábrahám et al. (2000) estimates. However, if the source has a disk, the escaping radiation field will be anisotropic, and if the disk is viewed close to edge-on, this would lead to the photometrically determined luminosity, and hence the expected dust sublimation radius, being underestimated, probably by a factor of approximately two to four. Such an underestimate is easily compatible with a small uncertainty in the spectral type.

Finally, if the NIR flux arises mostly at the inner edge of a disk, as in the models of Lachaume et al. (2003), Dullemond et al. (2001), and Natta et al. (2001), this region might well be obscured from view by the outer parts of any flaring disk or circumstellar torus. The observed flux would then be reflected from the circumstellar material, and the size of the dominant component in the NIR might well be considerably larger than the original dust sublimation radius. This possibility is of course entirely consistent with the high level of linear polarization observed from the object.

\subsection{The other components}

The largest structure seen at $H$ but not at $K^{\prime}$ clearly corresponds to the structure seen by Leinert et al. (1991) and also by Piirola et al. (1992) and others. This extended $H$-band component is caused by scattered light from the extended nebula.
This intermediate structure (100 mas $\sim 60$ AU in radius) may represent scattering from the flaring parts of the outer disk and probably corresponds to the bright knot seen in the HST images.

\section{Conclusions}

Our $H$ - and $K^{\prime}$-band speckle interferometric observations of V376 Cas suggest two resolved compact components, with characteristic radii of 100 mas and 8 mas. Additionally, a scattering envelope about $2^{\prime \prime}$ in diameter is seen in the $H$ band. The resolved components together account for at least $25 \%$ of the flux at $K^{\prime}$ and $35 \%$ of the flux at $H$. The smallest resolved structure may be related to the radius of dust sublimation at the inner part of a disk or envelope heated directly by stellar radiation. However, the actual size of this region ( 8 mas or $5 \mathrm{AU}$ ), is about six times too large for the supposed luminosity of V376 Cas. If V376 Cas possesses a disk, it is likely to be viewed close to edge-on, and this would lead to the photometrically determined luminosity being an underestimate of the true luminosity. Furthermore, the outer part of any disk probably obscures the inner disk edge, where the dust sublimation region would be situated. The NIR flux observed would then most likely arise from scattering from the inner edge of a cavity above the disk plane. This hypothesis is further strengthened by the high degree of linear polarization displayed by this object.

Acknowledgements. We thank John Monnier for providing us with data used in Fig. 3 and Sasha Men'shchikov and Thomas Driebe for helpful discussions. We also thank the referee, Peter Tuthill, whose comments have led to significant improvements in the manuscript.

\section{References}

Ábrahám, P., Leinert, C., Burkert, A., Henning, T., \& Lemke, D. 2000, A\&A, 354, 965

Adams, F. C., Lada, C. J., \& Shu, F. H. 1987, ApJ, 312, 788

Akeson, R. L., Ciardi, D. R., van Belle, G. T., Creech-Eakman, M. J. Lada, E. A. 2000, ApJ, 543, 313

Allen, D. A. 1972, ApJ, 172, L55

Asselin, L., Ménard, F., Bastien, P., Monin, J.-L. \& Rouan D. 1996, ApJ, 472, 349

Brandner, W., Grebel, E. K., Chu, Y.-H., et al. 2000, AJ, 119, 292

Chavarria, K. C. 1985, A\&A, 148, 317

Chiang, E. I., \& Goldreich, P. 1997, ApJ, 490, 368

Close, L. M., Roddier, F., Hora, J. L., et al. 1997, ApJ, 489, 210

Cohen, M. 1974, MNRAS, 169, 257

Cohen, M., \& Kuhi, L. V. 1979, ApJS, 41, 743

Corcoran, D., Ray, T. P., \& Bastien, P. 1995, A\&A, 293, 550

Danchi, W. C., Tuthill, P. G., \& Monnier, J. D. 2001, ApJ, 562, 440

Dullemond, C. P., Dominik, C., \& Natta, A. 2001, ApJ, 560, 957

Driebe, T. 2001, Ph.D. Thesis, University of Bonn

Hartmann, L., Kenyon, S. J., \& Calvet, N. 1993, ApJ, 407, 219

Herbig, G. H. 1960, ApJS, 4, 337

Hillenbrand, L. A., Strom, S. E., Vrba, F. J., \& Keene, J. 1992, ApJ, 397,613

Hofmann, K.-H., Balega, Y., Ikhsanov, N. R., Miroshnichenko, A. S., \& Weigelt, G. 2002, A\&A, 395, 891

Lachaume, R., Malbet, F., \& Monin, J.-L. 2003, A\&A, 400, 185

Labeyrie, A. 1970, A\&A, 6, 85

Leinert, C., Haas, M., \& Lenzen, R. 1991, A\&A, 246, 180 
Leinert, C., Haas, M., Ábrahám, P., \& Richichi, A. 2001, A\&A, 375, 927

Lynden-Bell, D., \& Pringle, J. E. 1974, MNRAS, 168, 603

Malbet, F., Berger, J.-P., Colavita, M. M., et al. 1998, ApJ, 507, L149

Malbet, F., Lachaume, R., \& Monin J.-L. 2001, A\&A, 379, 515

Mannings, V., \& Sargent, A. I. 1997, ApJ, 490, 792

Mannings, V., \& Sargent, A. I. 2000, ApJ, 529, 391

McCaughrean, M. J., \& O’Dell, C. R. 1996, AJ, 111, 1977

Millan-Gabet, R., Schloerb, P., \& Traub, W. A. 2001, ApJ, 546, 358

Miroshnichenko, A., Ivezić, Z., \& Elitzur, M. 1997, ApJ, 475, L41

Miroshnichenko, A., Ivezić, Z., Vinković, D., \& Elitzur, M. 1999, ApJ, 520, L115

Monin, J.-L., \& Bouvier, J. 2000, A\&A, 356, L75

Monnier, J. D., \& Millan-Gabet, R. 2002, ApJ, 579, 694

Natta, A., Palla, F., Butner, H. M., Evans, N. J., \& Harvey, P. M. 1992, ApJ, 391, 805

Natta, A., Palla, F., Butner, H. M., Evans, N. J., \& Harvey, P. M. 1993, ApJ, 406, 674
Natta, A., Prusti, T., Neri, R., et al. 2001, A\&A, 371, 186

Palla, F., \& Stahler, S. W. 1993, ApJ, 418, 414

Pezzuto, S., Strafella, F., \& Lorenzetti, D. 1997, ApJ, 485, 290

Piirola, V., Scaltriti, F., \& Coyne, G. V. 1992, Nature, 359, 399

Preibisch, T., Balega, Y. Y., Schertl, D., Smith, M. D., \& Weigelt, G. 2001, A\&A, 378, 539

Rodriguez, L. F., \& Canto, J. 1983, RMxAA, 8, 163

Schertl, D., Balega, Y., Hannemann, T., et al. 2000, A\&A, 361, L29

Tuthill, P. G., Monnier, J. D., \& Danchi, W. C. 2001, Nature, 409, 1012

Tuthill, P. G., Monnier, J. D., Danchi, W. C., Hale, D. D. S., \& Townes, C. H. 2002, ApJ, 577, 826

Weigelt, G., Balega, Y. Y., Hofmann, K.-H., \& Preibisch, T. 2002a, A\&A, 392, 937

Weigelt, G., Balega, Y. Y., Preibisch, T., Schertl, D., \& Smith, M. D. 2002b, A\&A 381, 905 